\title{
A Method of Reaching Extreme Altitudes.
}

By Robert H. Goddard, Professor of Physics, Clark College, Worcester, Mass.

$\mathrm{I}^{T}$ is the purpose of the present article to state the general principles and possibilities of the method of reaching great altitudes with multiple charge rockets, from which the exploded gases are ejected with high efficiency.

\section{Fundamental Principle.}

The basic idea of the method, briefly stated in general terms, is this: Given a mass of explosive material of as great energy content as possible, what height can be reached if a large fraction of this material is shot downwards, on exploding, with as high a speed as possible? It is evident, intuitively, that the height will be great if the fraction of material that remains is small and the velocity of ejection of the gases is high.

A theoretical treatment of the subject shows that, provided the speed of ejection of the gases is high, and the proportion of propellant is large, the initial masses necessary to raise a given mass to great heights are surprisingly small, but are enormously large if these conditions are not satisfied.

\section{Principles to be Applied in Practice.}

(I) In order to apply practically the general principle above stated, there are three conditions that must be realised experimentally: First, the gases produced by the explosion must be ejected downwards with the greatest efficiency possible. This requirement must be met by burning the explosive in a strong combustion chamber, to which a tapered nozzle is attached, in order to obtain the work of expansion of the gases.

The apparatus used in the first experiments is shown in Fig. $\dot{x}$, in which $\mathrm{P}$ is the charge of dense smokeless powder, and $\mathrm{B}$ is the wadding. Three steel plugs were used, to vary the size of the powder chamber. The velocity of the gases highest velocity being nearly 8000 ft.-sec., produced by the chamber shown in Fig. 2, whereas

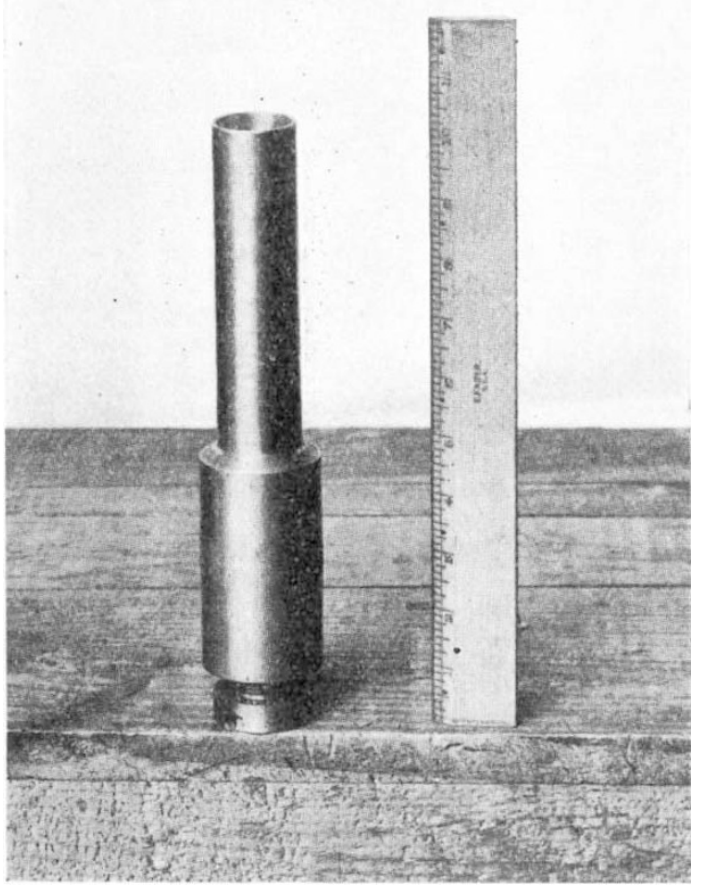

FIG, 2.-Chamber by which ejec ed gases were given a velocity of nearly $8000 \mathrm{ft}$. per sec.

for ordinary rockets the velocity is but Iooo ft.sec. Incidentally, the energy of motion of the gases in the case under discussion is more than 64 per cent. of the heat energy of the powder, whereas for ordinary rockets the efficiency thus defined is but 2 per cent.

An interesting way of emphasising the magnitude of the velocity, $8000 \mathrm{ft} .-\mathrm{sec}$., is to compare it with the "velocity of escape," or the "parabolic velocity" of planets. This velocity of escape is the velocity a body would require, pro- was measured by supporting the chamber in a ballistic pendulum, and observing the motion of the recoil.

It was found, by experiment, that the energy of motion of the ejected gases as compared with the heat energy of the powder could be increased very greatly over that for ordinary rockets, the NO. 2652 , VOL. IO5] jected upwards from a planet, in order to escape to infinity, and is a perfectly definite velocity, depending only upon the mass and diameter of the planet. For the moon the velocity is $\mathrm{I} \cdot 5$ miles per second, and for the planet Mars 3.0 miles per second. Thus if the chamber shown in Fig. 2 were placed upon the surface of the moon 
and fired, most of the gases would escape from the moon's attraction. The highest velocity gases would without doubt (since $8000 \mathrm{ft} .-\mathrm{sec}$. is only the average velocity) escape from Mars, if the planet had no atmosphere.

It should be remarked that, as shown by experimental results, the best form of nozzle has not yet been made, so that even $8000 \mathrm{ft}$. $-\mathrm{sec}$. can be exceeded by further research.

(2) The heavy chamber, as mentioned above, while permitting high velocities of the ejected gases to be obtained, would be an actual disadvantage if a single charge were to be fired,

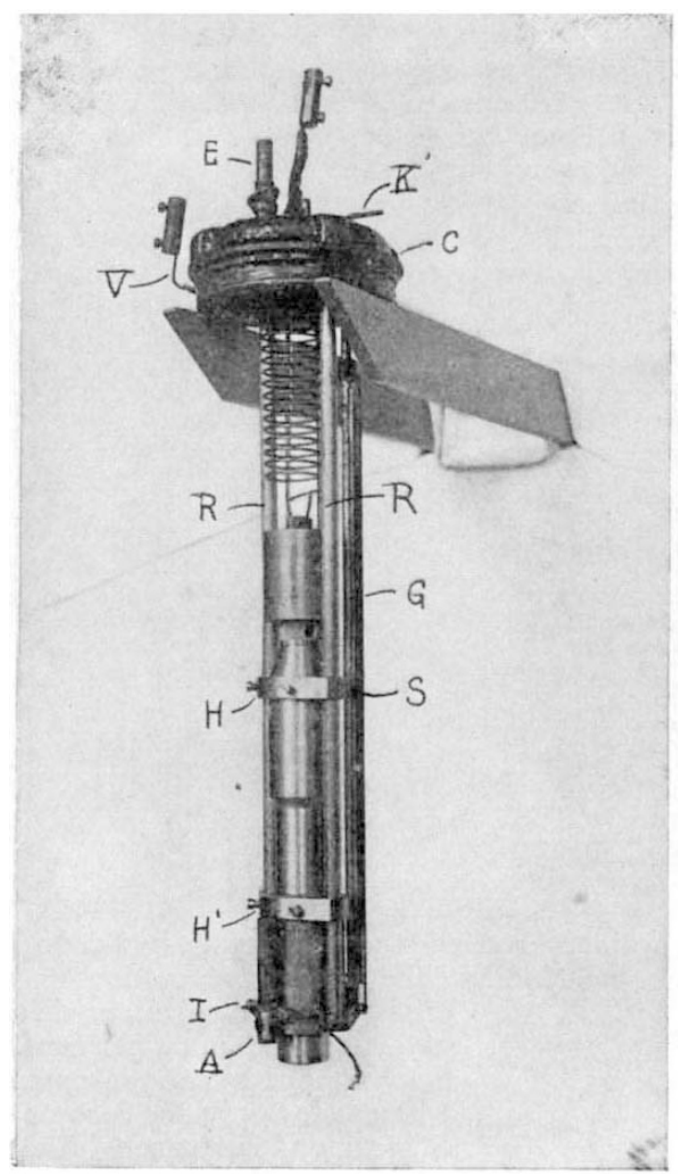

FIG. 3.-Chamber held in a support to test influence of air upon the propulsion of a rocket.

because of the large weight. It is necessary, then, that some means should be employed whereby charges may be fed successively into the same combustion chamber. If this is done it is evident that most of the rocket can consist of propellant, which is one of the conditions necessary for the attainment of great altitudes.

(3) When the magazine containing the charges just mentioned is nearly empty, it is easily seen that the propellant is no longer a large fraction of the entire mass of the apparatus. Hence, in order that the fraction shall remain large, it is NO. 2652 , VOL. IO5] necessary that one or more rockets, really copies in miniature of the larger primary rocket, should be used if the most extreme altitudes are to be reached, in order that the above fraction will, at no time during the ascent, become small.

\section{Summary of Results to Date.}

The theoretical work, done at Princeton University in rgr2, was not followed by experimental tests until I9I5, at Clark University. The work has since been continued at Clark University, in the magnetic laboratory at the Worcester Polytechnic Institute, and at the

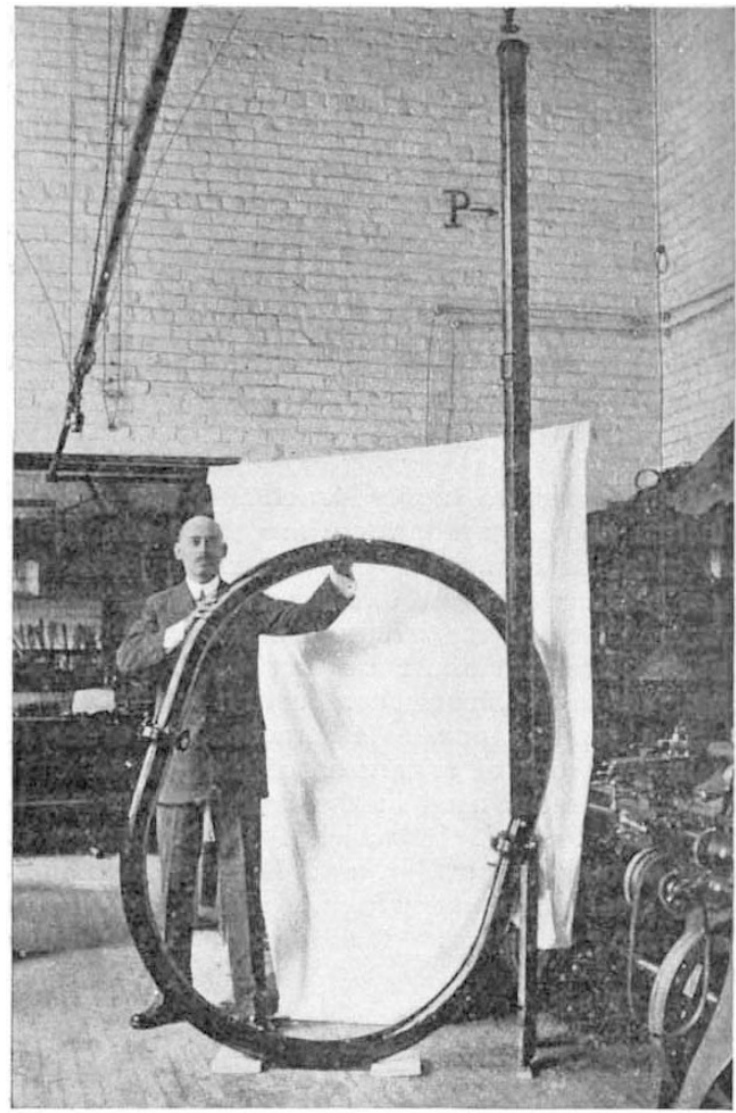

Fig. 4.-Pipe into which chamber was fired to a tank exhausted to a low pressure, the gases moving in a circular path until st spped by friction.

Mount Wilson Observatory in California-for the greater part of the time under a grant from the Smithsonian Institution.

The results of this work have shown, first, that most of the heat energy of even so powerful a propellant as dense nitroglycerine smokeless powder can be converted into kinetic energy of the ejected gases. They have demonstrated, secondly, that a multiple charge rocket can be made which will fire several charges in succession, is light and simple, and travels straight.

In order to demonstrate whether or not the rocket depended for propulsion upon the presence 
of air, a large number of experiments were performed in which the chamber, Fig. I, was held

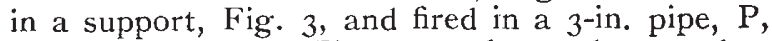
on a large tank, Fig. 4, exhausted to a low pressure. These experiments demonstrated that the presence of the air was not necessary for reaction and that the recoil is produced by reaction from the high-velocity gases that are ejected. The operation of the jet in vacuo need not appear mysterious if one thinks of the ejected gases as a charge of fine shot moving with a very high velocity. Obviously the chamber will react, or "kick," when this charge is fired,

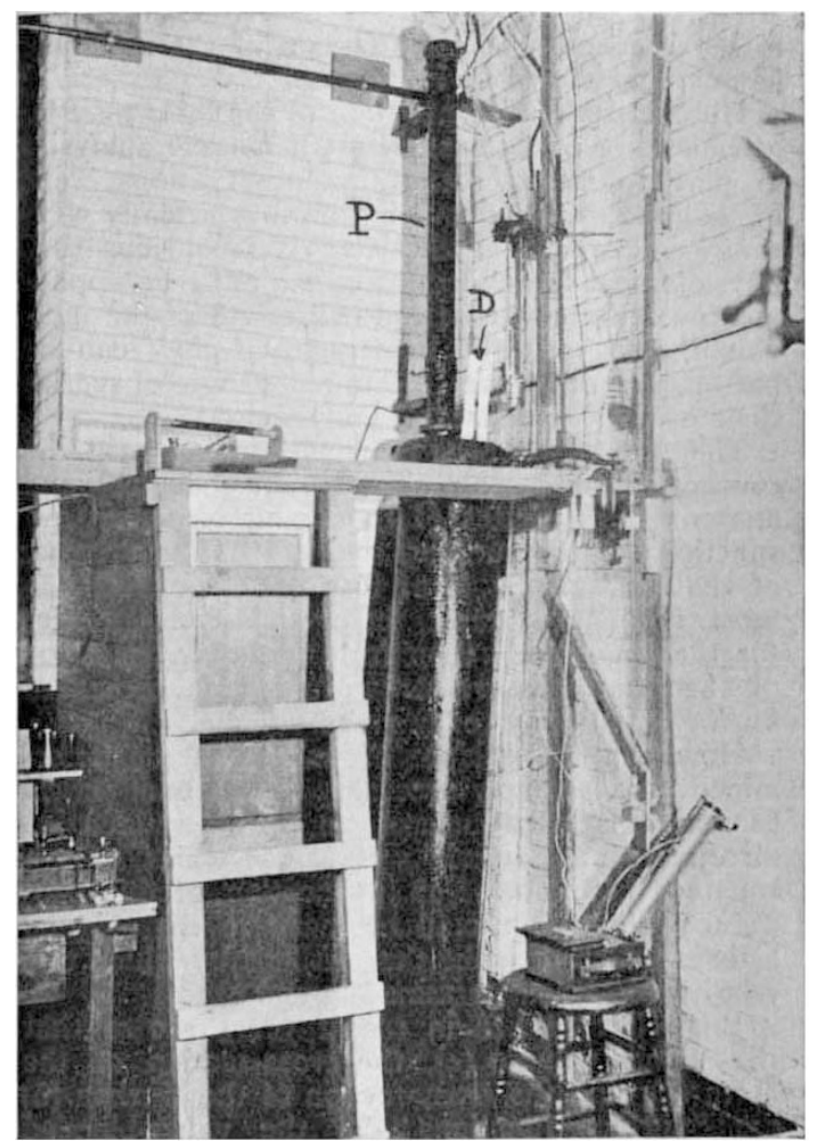

FIG. 5. - Tank in which the gases struck a coil of wire-fencing.

exactly as a shot-gun "kicks" when firing a charge of ordinary shot.

The gases were prevented from rebounding from the bottom of the tank, Fig. 4, by the form of the tank, the gases moving in a circular path until stopped by friction. Another tank, Fig. 5, was also used, in which rebound was prevented by the gases striking a large coil of $\frac{1}{2}$-in. mesh wire-fencing. The results with both tanks agreed down to the lowest pressure employed, $0.5 \mathrm{~mm}$. of mercury, which is probably the pressure that exists at a height of thirty miles.

NO. 2652 , VOL. IO5]
The figures given in the Smithsonian publication regarding the initial masses necessary to propel I lb. to various heights, such as $12.3 \mathrm{lb}$. for 430 miles, and $438 \mathrm{lb}$. for an "infinite" altitude (for the most favourable conditions, in so far as they are set forth in that publication), do. not assume a larger velocity of ejection of the gases than has been obtained experimentally, but do assume a greater lightness than has so far been obtained. No attempt has, however, been made to reduce any part of the apparatus to the minimum weight possible, and it is believed that with further research such lightness as is assumed is realisable.

At the present time, the work that is being done is the developing of a rocket, of small size, for employing a large number of cartridges, or charges, and this is being done on the remainder of the original grant from the Smithsonian Institution.

\section{Application of the Method.}

The most important of the immediate applications of the method is in the providing of a simple and, when sufficiently developed, inexpensive means of obtaining meteorological data at the Io-kilometre level. It is well recognised that this is the most important level for studying pressure, temperature, humidity, and wind velocity; and any means of sending recording instruments rapidly into this region, and of obtaining data soon after the ascent has been made, is certain to be of value in weather forecasting.

At greater elevations the study of temperature, pressure, wind velocity, and composition of the atmosphere is of scientific importance, and also the study of the aurora, during the day as well as at night, and the radiations from the sun that are otherwise absorbed by the atmosphere.

A further application of much general interest is the possibility of sending a mass beyond the predominating gravitational field of the earth. Concerning the possibility of demonstrating this point by hitting the moon with a rocket, it can be said, apart from the questions of aiming and of correcting the flight, that the ignition of but a few pounds of flash powder should be visible in a powerful telescope, provided, of course, that the conditions of ignition were substantially the same as those in certain experiments described in a recent Smithsonian publication, in which $\mathrm{I} / 20$ of a grain fired in vacuo was observed at a distance of $2 \frac{1}{4}$ miles.

Regarding these questions, as well as others which naturally follow, the writer believes that detailed discussion, before one has checked up matters completely by experiment, is unwise, for this merely precipitates a flood of useless argument, to which reply, in some form, must be made. The ideal method, which unfortunately is not always possible, is to solve a problem completely, as was done with the tests of the jet in vacuo, and then to state the results. 